\title{
Effect of updating training on fluid intelligence in children
}

\author{
ZHAO Xin ${ }^{1,2}$, WANG YiXue ${ }^{2}$ LIU DanWei ${ }^{2} \&$ ZHOU RenLai ${ }^{1,2 *}$ \\ ${ }^{1}$ Beijing Key Laboratory of Applied Experimental Psychology, School of Psychology, Beijing Normal University, Beijing 100875, China;
}

${ }^{2}$ State Key Laboratory of Cognitive Neuroscience and Learning, Beijing Normal University, Beijing 100875, China

Received March 23, 2011; accepted May 3, 2011

\begin{abstract}
Recent studies have indicated that working memory (WM) training can improve fluid intelligence. However, these earlier studies confused the impact of WM storage and central executive function on the effects of training. The current study used the running memory task to train the updating ability of 9-11 year-old children using a double-blind controlled design. The results revealed that children's fluid intelligence was significantly improved by memory-updating training. Overall, our findings suggest that the increase in fluid intelligence achieved with WM training is related to improving central executive function.
\end{abstract}

working memory updating, fluid intelligence, children

Citation: Zhao X, Wang Y X, Liu D W, et al. Effect of updating training on fluid intelligence in children. Chinese Sci Bull, 2011, 56: 2202-2205, doi: 10.1007/s11434- 011-4553-5

The question of whether human intelligence can be improved by training at a young age has long been a contentious issue in psychology research [1-3]. Recent studies have reported that fluid intelligence can be improved with working memory (WM) training [4,5]. Klingberg et al. [4] used a new type of WM training task to train children suffering from attention deficit hyperactivity disorder (ADHD) for $20 \mathrm{~d}$, with $20 \mathrm{~min}$ of training per day, for 4-6 d per week. This training included tasks involving visuospatial WM, backwards digit-span, letter-span, and choice reaction time. The results revealed that WM training improved WM ability in ADHD children, and ameliorated their clinical symptoms. Meanwhile, another study reported that WM training significantly improved the performance of children with ADHD on tests of intelligence. In addition, Jaeggi et al. [5] used a WM N-back task to train WM in adults. In their experiment, participants were randomly divided into 4 groups and trained for $8,12,17$ and $19 \mathrm{~d}$. The results revealed that, compared with a control group, the intelligence performance of participants who received WM training was significantly improved. In addition, a longer training duration was associated with greater improvements in

*Corresponding author (email: rlzhou@bnu.edu.cn) intelligence.

Several studies have reported that WM can be used to predict intelligence $[6,7]$. Based on these findings, the possibility of improving fluid intelligence with WM training has been investigated $[3,8]$. However, WM, considered the core of high-level human cognitive activity, is an extremely complex ability $[9,10]$. The studies by Klingberg et al. [4] and Jaeggi et al. [5] used the WM span task and the N-back task as training, respectively. The training in these tasks thus included not only a central executive component, but also a storage component $[11,12]$. However, the combination of these two components makes it difficult to determine which component of the WM training improves intelligence, because it is unclear whether the improvement is caused by expanding storage capacity or upgrading central executive function.

A growing number of studies have focused on the role of central executive functioning in WM when investigating the link between WM and intelligence [13-15]. However, it has been proposed that the functioning of working memory updating (WMU) has an important role in the relationship between WM and intelligence [16]. First, keeping track of targets, simultaneously processing stimuli, searching for new rules and filtering irrelevant information are crucial 
abilities that strongly affect intelligence performance [17], and the processing underlying these abilities is similar to the processing involved in WMU [18]. Second, the updating of WM regulates almost all of the changes in fluid intelligence related to aging, which acts as a mediating variable between age and fluid intelligence [19]. Third, some indirect evidence has demonstrated that the neural mechanism of WMU and fluid intelligence partially overlaps in the prefrontal cortex [14]. This evidence suggests that the improvement of intelligence through WM training is likely to be related to WMU. Updating is an essential component in the central executive component of WM, the major function of which is to monitor input information, replace old information that is irrelevant to the ongoing task with new information that is relevant to the ongoing task, and revise the content of memory continuously and simultaneously. The running memory task is widely used as an index of WMU. In this task, participants are presented with a series of unknown items of a certain length, which they are required to recall in order, within a certain length of time [20]. Importantly, the running memory task purely reflects the central executive functioning involved in WM, removing the potential confounding effects of storage. Van der Linden and colleagues [21] reported that the storage function of healthy elderly participants was intact, even when their performance on the running memory task was impaired. Mirris and John [20] used the running memory task to interfere with the phonological loop. Their results revealed that interference did not affect the updating of the phonological loop, which proved that running memory task reflects the central executive ability not the storage function. Thus, the present study used the running memory task to train WMU ability, enabling us to investigate whether training WMU specifically could directly promote the improvement of fluid intelligence.

We recruited 33 (19 male, 14 female) participants from an elementary school in Beijing. All participants were in 4th grade, ranging from 9 to 11 years of age (average 9.76 years, standard deviation 0.61). All participants had normal vision, hearing and intelligence. Participants were randomized into a training group (16 participants, 7 male, 9 female) and a control group (17 participants, 12 male, 5 female) based on their student identification numbers. We matched the two groups for intelligence to ensure homogeneity between experimental conditions using Raven's Standard Progressive Matrices. These data were used as pre-test scores. Independent $t$-tests (2-tailed) did not reveal any significant differences between the two groups in age or intelligence using. None of the children had previously participated in similar experiments.

Our training task was an adapted version of the running memory paradigm described by Collette et al. [18] and Mirris et al. [20]. The program was compiled in Visual C\#. This paradigm involves 2 tasks, as follows. (i) The animal running memory task: first, a fixation " + " was presented in the center of the screen to indicate the onset of the task. Several images of animals were then presented one after the other, for $1750 \mathrm{~ms}$ each. The number of animal stimuli presented varied between different trials, with $5,7,9$ or 11 images presented in each trial. Every trial type occurred 5 times, in a random order, so that the total number of presentations was 20. Participants were required to sequentially remember the last 3 animals presented. (ii) Cartoon location running memory task: first, a fixation "+" was presented in the center of the screen to indicate task onset. A 9-square grid was then presented in the center of the screen, containing an image of a cartoon face (the Nintendo character "Mario"), which could occur in any of the 9 squares, appearing for a duration of $1750 \mathrm{~ms}$. The number of times the face was presented varied between different trials, such that there were 4 trial types, involving 5, 7, 9 or 11 presentations. Every trial type was presented 5 times at random, so that the total number of presentations was 20. Participants were required to sequentially remember the locations of the last 3 squares in which the character was presented. Lastly, in the center of the screen there will be 39 -square grids and participants need to sequentially click the corresponding positions of the 3 grids to the latest 3 positions separately. After finishing one trial, participants would confirm their selection by clicking "sure" to begin the next trial. Correct responses were recorded as a score of " 1 ", meaning that the total score was 20. When the answer was correct, a smiley-face figure was presented at the bottom of the screen as feedback. When the response was incorrect, a bomb figure appeared in the same location. The feedback was presented every trial, and participants received rewards according to their scores. To avoid possible placebo effects and the effects of other irrelevant interference variables (e.g., familiar experimenter and environment), we used a double-blind controlled design. Thus, the experimenters conducting the pre- and post-test phases were not present during the training phase. All 33 participants completed the pre- and post-test phases in the same room of the school. In addition, we minimized distracting sounds that could interrupt the participants' attention during the test. A period of $30 \mathrm{~d}$ was interpolated between the pre- and post-test phases. In the training phase, the training group performed the running memory task in the computer room of the school, and received 15-20 min training every day, for 3-4 d per week, for 4 weeks. This resulted in a total of 15 training days. The control group performed irrelevant computer games, in the same room.

SPSS13.0 was used for data aggregation and statistical analysis, and the training effects were examined using one-way repeated measures analysis of variance (ANOVA). The results are shown in Figures 1 and 2. The results revealed that training time significantly affected scores in the animal running memory task $(F(14,210)=17.300, P<$ $\left.0.001, \eta^{2}=0.536\right)$, such that a greater number of training days was associated with a significant increase in test score. In addition, the amount of training also significantly affected 


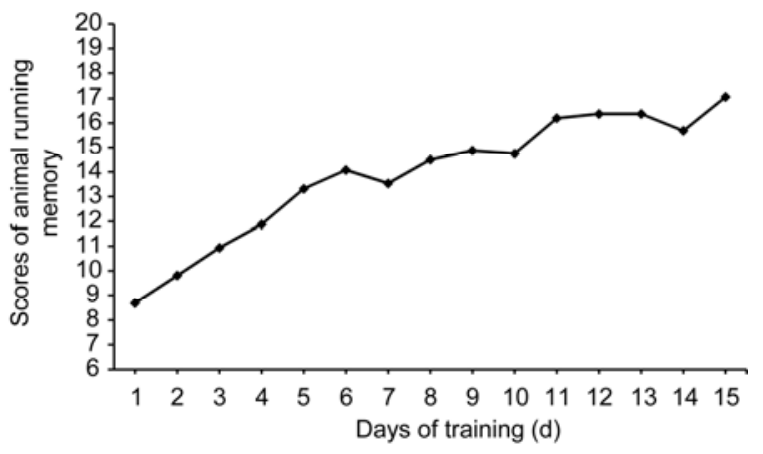

Figure 1 Improvement during training with the animal running memory task.

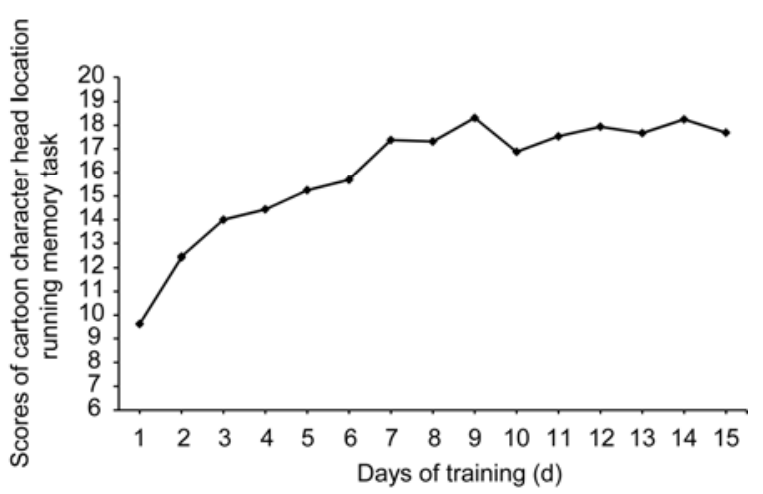

Figure 2 Improvement during training on the cartoon location running memory task.

scores in the cartoon location running memory task $(F(14$, 210)=17.199, $\left.P<0.001, \eta^{2}=0.534\right)$, such that higher scores were significantly associated with more training.

For the Raven's Standard Progressive Matrices test scores, we subtracted the pre-test scores from the post-test scores, then calculated the mean values of both the training and control groups. Independent-samples $t$-tests were used to examine the additive values (i.e., the effects of WMU training on children's fluid intelligence). Table 1 lists the mean values and additive values of Raven's Standard Progressive Matrices test scores in the pre-and post-test phases for both the training and control groups.

The analysis of the additive mean values revealed that fluid intelligence scores in the training group were significantly higher than those in the control group, $t(31)=2.271$, $P<0.05$, Cohen's $d=0.98$. To further examine the causality of WMU, and allow comparison with the findings of

Table 1 Raven's Standard Progressive Matrices test scores in pre- and post-test phases in the training and control groups, and the additive values $(M \pm \mathrm{SD})$

\begin{tabular}{cccc}
\hline & Pre-test & Post-test & Additive value \\
\hline $\begin{array}{c}\text { Training group } \\
(n=16)\end{array}$ & $42.13 \pm 6.12$ & $45.94 \pm 3.45$ & $3.81 \pm 4.59$ \\
$\begin{array}{c}\text { Control group } \\
(n=17)\end{array}$ & $40.41 \pm 5.14$ & $41.12 \pm 5.06$ & $0.71 \pm 3.18$ \\
\hline
\end{tabular}

Klingberg et al. [4], we analyzed the correlation between the degree of improvement in WMU ability (pre-test/posttest running memory task scores) and that of the fluid intelligence (pre-test/post-test Raven's Standard Progressive Matrices scores). The results revealed a significant correlation $(r=0.54, P<0.05)$.

The current study used a double-blind controlled design to train WMU ability in children aged between 9 and 11 years old. The results revealed that after a 4-week period involving $15 \mathrm{~d}$ of training with a running memory task, children's fluid intelligence test performance was significantly improved. In contrast with previous studies [4,5], the present study adopted a training task that is considered to purely involve central executive WM only. The use of this training task thus avoided the effects of storage ability in training, demonstrating that WMU ability can influence fluid intelligence. These results support the notion that WM is a fundamental component of children's fluid intelligence development [19]. The attention control model of WM $[7,14,22]$ postulates that the major performance of the relationship between WM and fluid intelligence is a requirement of the control of attention, and that attentional control is completed by the central executive component of WM. The current study used a running memory task to train children's WMU ability, revealing that the scores on the training task increased with the amount of training. Moreover, children's fluid intelligence was also found to be significantly improved after training. It is possible that the training period promoted the development of children's attentional control, increasing their ability to maintain the activation of information relevant to the target while blocking the maintenance of irrelevant information, which is crucial for fluid intelligence [17]. Second, the brain areas involved in WMU and fluid intelligence overlapped in the prefrontal cortex $[11,14,23]$. Furthermore, two studies by Westerberg et al. $[24,25]$ revealed that WM training strengthened activation in certain brain areas related to WM. This strengthening did not occur in areas that were not activated before training, suggesting that this type of cortical activation reflects the reinforcement of initial activation rather than the recruitment of new areas. Thus, training WMU ability may promote the development of children's prefrontal cortex function, causing a transferable effect that can lead to the improvement of fluid intelligence. In addition, $\mathrm{McNab}$ et al. [26] reported that the density of dopamine D1 receptors in the cortex changed with WM training. WM and fluid intelligence are both dependent on the presence of dopamine transmitters and the plasticity of the dopamine D1 receptor system. Taken together with this previous finding, the current results suggest that WM training may change the density of dopamine D1 receptors in the prefrontal cortex of children, further promoting the ability to transfer learning. Future studies should use techniques from cognitive neuroscience, including event-related potentials, functional magnetic resonance imaging, and positron emission tomography, 
to determine the underlying causes of the effects of training WMU on fluid intelligence, and explore the plasticity of human cognitive functions.

We thank the President Liu Tong of XinGong Primary School and all over the teachers and students who joined the research. This work was supported by the National Basic Research Program of China (2011CB711000) and the Program for Changjiang Scholars and Innovative Research Team in University (IRT0710).

\section{Zhou R L, Zhao X. Chin Soc Sci Today, 2010-05-06, 4}

Moody D E. Intelligence, 2009, 37: 327-328

3 Sternberg R J. Proc Natl Acad Sci USA, 2008, 105: 6791-6792

4 Klingberg T, Forssberg H, Westerberg H. J Clin Exp Neuropsyc, 2002, 24: 781-791

5 Jaeggi S M, Buschkuehl M, Jonides J, et al. Proc Natl Acad Sci USA, 2008, 105: 6829-6833

6 Zhao X, Zhou R L. J Beijing Norm Univ (Soc Sci), 2010, (5): 38-44

7 Engle R W. Curr Anthropol, 2010, 51: 17-26

8 Zhao X, Zhou R L. Adv Psychol Sci, 2010, (5): 38-44

9 Baddeley A D. Science,1992, 255: 556-559
10 Baddeley A D. Nat Rev Neurosci, 2003, 4: 829-839

11 Owen A M, Mcmillan K M, Laird A R, et al. Hum Brain Mapp, 2005, 25: 46-59

12 Conway A, Kane M J, Bunting M F, et al. 2005, 12: 769-786

13 Engle R W, Tuholski S W, Laughlin J E, et al. J Exp Psychol Gen, 1999, 128: 309-331

14 Kane M J, Engle R W. Psych Bull Rev, 2002, 9: 637-671

15 Unsworth N, Engle R W. Intelligence, 2005, 33: 67-81

16 Friedman N P, Miyake A, Corley R P, et al. Psychol Sci, 2006, 17: 172-179

17 Carpenter P A, Just M A, Shell P. Psychol Rev, 1990, 97: 404-431

18 Collette F, Van der Linden M. Neurosci Biobehav R, 2002, 26: $105-125$

19 Chen T Y, Li D M. Aging Neuropsychol Cogn, 2007, 14: 631-646

20 Mirris N, John D M. Br J Psychol, 1990, 81: 111-121

21 Van der Linden M, Brédart S, Beerten A. Br J Psychol, 1994, 85: 145-152

22 Conway A R A, Cowan N, Bunting M F, et al. Intelligence, 2002, 30: 163-183

23 Jung R E, Haier R J. Behav Brain Sci, 2007, 30: 135-187

24 Westerberg H, Klingberg T. Physiol Behav, 2007, 92: 186-192

25 Olesen P J, Westerberg H, Klingberg T. Nat Neurosci, 2004, 7: 75-79

26 McNab F, Varrone A, Farde L, et al. Science, 2009, 323: 800-802

Open Access This article is distributed under the terms of the Creative Commons Attribution License which permits any use, distribution, and reproduction in any medium, provided the original author(s) and source are credited. 